\title{
Early immune responses and prognostic factors in children with COVID-19: a single- center retrospective analysis
}

Wenjie Lu ${ }^{1 \dagger}$, Li Yang ${ }^{1 \dagger}$, Xiong Li ${ }^{2,3+}$, Ming Sun ${ }^{1}$, Aiping Zhang ${ }^{1}$, Shanshan Qi ${ }^{1}$, Zhi Chen ${ }^{1}$, Lannan Zhang ${ }^{1}$, Jianxin $\mathrm{Li}^{1}$ and Hao Xiong ${ }^{1^{*}}$

\begin{abstract}
Background: Early diagnostic indicators and the identification of possible progression to severe or critical COVID-19 in children are unknown. To investigate the immune characteristics of early SARS-CoV-2 infection in children and possible key prognostic factors for early identification of critical COVID-19, a retrospective study including 121 children with COVID-19 was conducted. Peripheral blood lymphocyte subset counts, T cell-derived cytokine concentrations, inflammatory factor concentrations, and routine blood counts were analyzed statistically at the initial presentation.

Results: The T lymphocyte subset and natural killer cell counts decreased with increasing disease severity. Group III (critical cases) had a higher Th/Tc ratio than groups I and II (common and severe cases); group I had a higher B cell count than groups II and III. IL-6, IL-10, IFN- $\gamma$, SAA, and procalcitonin levels increased with increasing disease severity. Hemoglobin concentration, and RBC and eosinophil counts decreased with increasing disease severity. Groups II and III had significantly lower lymphocyte counts than group I. T, Th, TC, IL-6, IL-10, RBC, and hemoglobin had relatively high contribution and area under the curve values.

Conclusions: Decreased T, Th, TC, RBC, hemoglobin and increased IL-6 and IL-10 in early SARS-CoV-2 infection in children are valuable indices for early diagnosis of severe disease. The significantly reduced Th and Tc cells and significantly increased IL-6, IL-10, ferritin, procalcitonin, and SAA at this stage in children with critical COVID-19 may be closely associated with the systemic cytokine storm caused by immune dysregulation.
\end{abstract}

Keywords: SARS-CoV-2, Children, Severe case, Lymphocyte subset, Inflammatory factor

\footnotetext{
* Correspondence: Xionghao_wch@sina.com

†'Wenjie Lu, Li Yang and Xiong Li contributed equally and share first authorship.

'Department of Hematology, Wuhan Children's Hospital (Wuhan Medical

Care Center for Women and Children), Tongji College, Huazhong University of Science and Technology, Wuhan, Hubei, China

Full list of author information is available at the end of the article
}

(c) The Author(s). 2021 Open Access This article is licensed under a Creative Commons Attribution 4.0 International License, which permits use, sharing, adaptation, distribution and reproduction in any medium or format, as long as you give appropriate credit to the original author(s) and the source, provide a link to the Creative Commons licence, and indicate if changes were made. The images or other third party material in this article are included in the article's Creative Commons licence, unless indicated otherwise in a credit line to the material. If material is not included in the article's Creative Commons licence and your intended use is not permitted by statutory regulation or exceeds the permitted use, you will need to obtain permission directly from the copyright holder. To view a copy of this licence, visit http://creativecommons.org/licenses/by/4.0/ The Creative Commons Public Domain Dedication waiver (http://creativecommons.org/publicdomain/zero/1.0/) applies to the data made available in this article, unless otherwise stated in a credit line to the data. 


\section{Introduction}

The coronavirus disease 2019 (COVID-19) outbreak in Wuhan, Hubei Province, China, in late December 2019 has been controlled by effective measures. However, the epidemic outside China has not been controlled effectively. Compared with adult patients, most children are infected with severe acute respiratory syndrome coronavirus 2 (SARS-CoV-2) through family clusters, with relatively mild clinical symptoms and a good prognosis [1]. Several studies have suggested that, in adults infected with SARS-CoV-2, the progression to severe cases is clearly associated with cytokine storm or cytokine storm syndrome, which is life-threatening and clinically characterized by systemic inflammation, hyperferritinemia, hemodynamic instability and multiple organ failure, and it is an important factor contributing to the lifethreatening clinical manifestations of H7N9, H5N1, and SARS [2-4]. To date, there has been no report on the indications for early diagnosis and identification of possible progression to severe or critical cases in children with SARS-CoV-2 infection.

We retrospectively collected the clinical data of 121 children with confirmed and highly suspected SARSCoV-2 infection who had been admitted to Wuhan Children's Hospital from January 25, 2020, to February 20, 2020, and analyzed the differences in the laboratory indices of peripheral blood from children with early mild and severe SARS-CoV-2 infection. We investigated the differences in the immune and stress responses in children with different disease severity in the early stage of SARS-CoV-2 infection, and aimed to provide a laboratory basis for early detection of disease progression and deterioration and effective clinical intervention in children with SARS-CoV-2 infection.

\section{Materials and methods General information}

The information on the laboratory test indices on initial admission, treatment, and prognosis of 121 children with confirmed and suspected SARS-CoV-2 infection who had been admitted to Wuhan Children's Hospital from January 25, 2020, to February 20, 2020, was collected, compiled, and retrospectively analyzed. The indices included: peripheral blood cell counts (white blood cells, red blood cells, neutrophils, lymphocytes, eosinophils), acute infection indices (ferritin [FERR], serum amyloid A [SAA], C-reactive protein [CRP], calcitonin [PCT]), cytokines (interleukin-2 [IL-2], IL-4, IL-6, IL-10, tumor necrosis factor alpha [TNF- $\alpha$ ], interferon gamma [IFN$\gamma]$ ), and lymphocyte subsets $\left(\mathrm{CD}^{+} \mathrm{T}\right.$ cells, $\mathrm{CD}^{+} \mathrm{CD} 4^{+} \mathrm{T}$ helper [Th] cells, $\mathrm{CD}^{+} \mathrm{CD}^{+}$cytotoxic $\mathrm{T}[\mathrm{Tc}]$ cells, $\mathrm{CD}_{16}{ }^{+} \mathrm{CD} 56^{+}$natural killer $[\mathrm{NK}]$ cells, $\mathrm{CD} 19^{+} \mathrm{B}$ cells, $\mathrm{CD} 4^{+} \mathrm{CD} 25^{+} \mathrm{T}$ regulatory [Treg] cells).
All children gave written informed consent signed by their legal guardians before being tested.

\section{Diagnostic criteria}

According to the recommendations for the diagnosis, prevention, and control of COVID-19 in children (first interim edition) [5], the children were diagnosed with suspected SARS-CoV-2 infection if both of the following criteria were met:

(1) Epidemiological history: History of travel or residence in Wuhan within 14 days before disease onset; or contact with patients with fever and respiratory symptoms or clusters of SARS-CoV-2 infection (in Hubei Province) within 14 days before disease onset.

(2) Clinical manifestations: a) Fever, fatigue, and dry cough (some children may have no or low fever); b) possible radiological features of pneumonia such as multiple ground-glass opacities and/or infiltrative shadows in the lungs; c) normal or decreased total white blood cell count, or decreased lymphocyte count in the early stage of onset; and d) clinical manifestations that cannot be fully explained by other etiologies.

Children with suspected SARS-CoV-2 infection were confirmed if they tested positive for SARS-CoV-2 nucleic acid in nasopharyngeal or throat swabs, and lower respiratory tract secretions by real-time fluorescence RT-PCR.

The infected children can be divided into five categories [5], as follows:

(1) Asymptomatic infection: The children have no clinical symptoms and signs, the chest imaging examination are normal, but the $2019 \mathrm{nCoV}$ nucleic acid test is positive, or the serum specific antibody are positive by the retrospective diagnosis.

(2) Mild: The children mainly have acute upper respiratory tract infection, including fever, fatigue, myalgia, cough, sore throat, runny nose and sneezing symptoms. Pharyngeal congestion can be seen in physical examination, but lungs have no positive signs. Some children may have no fever, but only with nausea, vomiting, abdominal pain, diarrhea or other gastrointestinal symptoms.

(3) Common type: The children are manifested as pneumonia. They often have fever and cough, initially mostly dry cough, then sputum cough, and some can have wheezing but no obvious shortness of breath and other hypoxia. Coarse rales, dry rales and/or wet rales can be heard from the lungs. Some of the children did not have any clinical symptoms 
and signs, but chest CT showed pulmonary lesions, which were subclinical.

(4) Severe type: Early respiratory symptoms such as fever and cough can be accompanied by digestive symptoms such as diarrhea. The disease usually progresses in about 1 week with dyspnea, central cyanosis, saturation of pulse oximetry $<0.92$ without oxygen inhalation and other hypoxia manifestations.

(5) Critical type: The children may rapidly progress to acute respiratory distress syndrome (ARDS) or respiratory failure, and may also develop multiple organ dysfunction such as shock, encephalopathy, myocardial injury or heart failure, coagulation dysfunction and acute kidney injury, which can be life-threatening.

\section{Disease classification}

According to the above criteria, the disease was divided into mild (group I: including asymptomatic infection, and mild and common cases), severe (group II), and critical cases (group III).

\section{Laboratory examinations}

According to the regulations of the local center for disease prevention and control, RT-PCR was used for SARS-CoV-2 nucleic acid testing [6]. RNA from the respiratory specimens was extracted with the High Pure Viral Nucleic Acid Kit (Zhongzhi, Wuhan, China). Lymphocyte subsets and cytokines were tested using a BD FACSCanto II flow cytometer (BD Biosciences). Lymphocytes were analyzed using a BD Multitest IMK Kit (cat. no. 340503, BD Biosciences); plasma cytokines (IL-2, IL-4, IL-6, IL-10, TNF- $\alpha$, IFN- $\gamma$ ) were determined using a human Th1/2 cytokine kit II (BD Biosciences).

\section{Statistical analysis}

Categorical variables are expressed as frequencies or percentages and were analyzed using the chi-square or Fisher exact test. Continuous variables are expressed as the mean \pm standard deviation. Differences between the three groups were compared using analysis of variance. Non-parametric variables are expressed as medians and interquartile ranges (IQRs), and significance was determined using the Mann-Whitney $U$ test and KruskalWallis test. Correlation coefficients of continuous variables were determined using the Spearman rank correlation test. The principal clinical parameters that could be used to distinguish mild infection from severe SARS$\mathrm{CoV}-2$ infection were determined using principal component analysis (PCA). The selected parameters for mild and severe SARS-CoV-2 infection were evaluated using the receiver operating characteristic (ROC) curve and the area under the ROC curve (AUC). $P<0.05$ was considered statistically significant. Tables 1,2 show the specific test indices and results. All statistical analyses were conducted using SPSS Version 22.0 software (SPSS Inc), $R$ package (v.3.6.1), and GraphPad Prism 8.0 (GraphPad Software LLC) (Tables 1, 2).

\section{Results \\ Demographics and clinical feature analysis of the children with COVID-19}

During the study period, a total of 490 children with confirmed or suspected COVID-19 were hospitalized at our hospital; among them, 121 children with complete clinical data were included in this study. The median age was 6.0 years (range: $0.13-15.0$ years). There were 82 boys $(67.8 \%)$ and 39 girls (32.2\%). There were 101 mild (83.5\%, asymptomatic infection, and mild and common cases), 12 severe (9.9\%), and 8 critical (6.6\%) cases.

Upon admission, the children's symptoms mainly included fever (78 cases, 64.5\%) and cough (69 cases, 57.0\%); minor symptoms included gastrointestinal symptoms (13 cases, 10.7\%), nasal discharge (11 cases, 9.1\%), sputum (11 cases, 9.1\%), fatigue (9 cases, 7.4\%), respiratory distress ( 9 cases, 7.4\%), headache (6 cases, 5.0\%), chest distress (5 cases, 4.1\%), and muscle soreness (4 cases, 3.3\%). Eighty-five patients (70.2\%) tested positive for SARS-CoV-2 nucleic acid on admission; 97 patients (80.2\%) had radiological changes in the lungs, with typical radiological changes of ground-glass opacities in both lungs; 85 children (70.2\%) were confirmed cases, and 36 children $(29.8 \%)$ were suspected cases.

The clinical symptoms of the children in groups I and II were relieved after treatment, and all were discharged after meeting the criteria of cure. The median lengths of hospital stay of the children in groups I and II were 11.0 days (IQR: $9.0-14.0$ days) and 10.0 days (IQR: $8.75-12.5$ days), respectively. The length of hospital stay between the two groups was not significantly different $(P=0.9)$. As of March 16, 2020, 2 patients in group III were still hospitalized, and 1 patient in group III died 36 days after admission, with a median hospital stay of 20.5 days (IQR: 17.25-35.25 days). There was no progression from mild to severe or from severe to critical cases among the hospitalized children.

\section{Analysis of the peripheral blood indices of the children infected with SARS-CoV-2}

The Kruskal-Wallis test and Spearman correlation analysis of the initial laboratory examinations on admission (Table 2, Fig. 1) of the 121 children showed that there was a significant decrease from groups I to III in $\mathrm{CD}^{+} \mathrm{CD}^{+}$Th cell $(P<0.001), \mathrm{CD}^{+} \mathrm{CD}^{+}$Tc cell $(P<$ $0.001)$, and $\mathrm{CD} 16^{+} \mathrm{CD} 56^{+} \mathrm{NK}$ cell $(P=0.001)$ counts. Groups II and III had significantly lower $\mathrm{CD} 19^{+} \mathrm{B}$ cell counts than group I $(P=0.026)$. Group III had a 
Table 1 Baseline Characteristics of Patients with SARS-CoV-2

\begin{tabular}{|c|c|c|c|c|c|}
\hline Baseline variables & Overall $(N=121)$ & Mild $(N=101)$ & Severe $(N=12)$ & Critical $(N=8)$ & $P$ Value \\
\hline \multicolumn{6}{|l|}{ Age (year) } \\
\hline Mean (SD) & $6.25(4.31)$ & $6.51(4.43)$ & $6.36(3.08)$ & $2.71(2.96)$ & 0.054 \\
\hline Median [Min, Max] & $6.00[0.130,15.0]$ & $6.75[0.130,15.0]$ & $6.96[1.00,10.6]$ & $1.67[0.170,8.00]$ & \\
\hline \multicolumn{6}{|l|}{ Gender } \\
\hline Male & 82 (67.8\%) & 65 (64.4\%) & 11 (91.7\%) & $6(75.0 \%)$ & 0.145 \\
\hline Female & 39 (32.2\%) & $36(35.6 \%)$ & $1(8.3 \%)$ & $2(25.0 \%)$ & \\
\hline \multicolumn{6}{|l|}{ PCR } \\
\hline No & $36(29.8 \%)$ & $24(23.8 \%)$ & 9 (75.0\%) & $3(37.5 \%)$ & 0.001 \\
\hline Yes & 85 (70.2\%) & 77 (76.2\%) & $3(25.0 \%)$ & $5(62.5 \%)$ & \\
\hline \multicolumn{6}{|l|}{ Imaging changes } \\
\hline No & 24 (19.8\%) & $22(21.8 \%)$ & $2(16.7 \%)$ & $0(0 \%)$ & 0.317 \\
\hline Yes & 97 (80.2\%) & 79 (78.2\%) & 10 (83.3\%) & $8(100 \%)$ & \\
\hline \multicolumn{6}{|l|}{ Dyspnoea } \\
\hline No & $112(92.6 \%)$ & 98 (97.0\%) & 11 (91.7\%) & $3(37.5 \%)$ & $<0.001$ \\
\hline Yes & $9(7.4 \%)$ & $3(3.0 \%)$ & $1(8.3 \%)$ & $5(62.5 \%)$ & \\
\hline \multicolumn{6}{|l|}{ Cough } \\
\hline No & $52(43.0 \%)$ & $43(42.6 \%)$ & $6(50.0 \%)$ & $3(37.5 \%)$ & 0.841 \\
\hline Yes & 69 (57.0\%) & 58 (57.4\%) & $6(50.0 \%)$ & $5(62.5 \%)$ & \\
\hline \multicolumn{6}{|l|}{ Fever } \\
\hline No & 43 (35.5\%) & 43 (42.6\%) & $0(0 \%)$ & $0(0 \%)$ & 0.001 \\
\hline Yes & 78 (64.5\%) & $58(57.4 \%)$ & 12 (100\%) & $8(100 \%)$ & \\
\hline \multicolumn{6}{|c|}{ Gastrointestinal symptoms } \\
\hline No & $108(89.3 \%)$ & $93(92.1 \%)$ & $10(83.3 \%)$ & $5(62.5 \%)$ & 0.027 \\
\hline Yes & $13(10.7 \%)$ & $8(7.9 \%)$ & $2(16.7 \%)$ & $3(37.5 \%)$ & \\
\hline \multicolumn{6}{|l|}{ Runny nose } \\
\hline No & $110(90.9 \%)$ & $91(90.1 \%)$ & $11(91.7 \%)$ & $8(100 \%)$ & 0.641 \\
\hline Yes & $11(9.1 \%)$ & $10(9.9 \%)$ & $1(8.3 \%)$ & $0(0 \%)$ & \\
\hline \multicolumn{6}{|l|}{ Headache } \\
\hline No & $115(95.0 \%)$ & 95 (94.1\%) & $12(100 \%)$ & $8(100 \%)$ & 0.535 \\
\hline Yes & $6(5.0 \%)$ & $6(5.9 \%)$ & $0(0 \%)$ & $0(0 \%)$ & \\
\hline \multicolumn{6}{|l|}{ Chest tightness } \\
\hline No & $116(95.9 \%)$ & $96(95.0 \%)$ & $12(100 \%)$ & $8(100 \%)$ & 0.597 \\
\hline Yes & $5(4.1 \%)$ & $5(5.0 \%)$ & $0(0 \%)$ & $0(0 \%)$ & \\
\hline \multicolumn{6}{|l|}{ Sputum production } \\
\hline No & $110(90.9 \%)$ & 91 (90.1\%) & $11(91.7 \%)$ & $8(100 \%)$ & 0.641 \\
\hline Yes & $11(9.1 \%)$ & $10(9.9 \%)$ & $1(8.3 \%)$ & $0(0 \%)$ & \\
\hline \multicolumn{6}{|l|}{ Muscle ache } \\
\hline No & $117(96.7 \%)$ & $98(97.0 \%)$ & $11(91.7 \%)$ & $8(100 \%)$ & 0.533 \\
\hline Yes & $4(3.3 \%)$ & $3(3.0 \%)$ & $1(8.3 \%)$ & $0(0 \%)$ & \\
\hline \multicolumn{6}{|l|}{ Fatigue } \\
\hline No & $112(92.6 \%)$ & $94(93.1 \%)$ & $11(91.7 \%)$ & $7(87.5 \%)$ & 0.84 \\
\hline Yes & $9(7.4 \%)$ & $7(6.9 \%)$ & $1(8.3 \%)$ & $1(12.5 \%)$ & \\
\hline \multicolumn{6}{|l|}{ With other diseases } \\
\hline No & $113(93.4 \%)$ & 98 (97.0\%) & $10(83.3 \%)$ & $5(62.5 \%)$ & $<0.001$ \\
\hline Yes & $8(6.6 \%)$ & $3(3.0 \%)$ & $2(16.7 \%)$ & $3(37.5 \%)$ & \\
\hline
\end{tabular}


Table 2 Peripheral blood indices' Results of children with SARS-COV-2

\begin{tabular}{|c|c|c|c|c|c|}
\hline Variables & Overall & Mild & Severe & Critical & $P$ Value \\
\hline Routine Blood Result & $(n=120)$ & $(n=101)$ & $(n=12)$ & $(n=7)$ & \\
\hline Hemoglobin, Mean (SD), g/L & $124(14.6)$ & $126.5(13.5)$ & $119.8(9.1)$ & $100.0(13.7)$ & $<0.001$ \\
\hline Platelet, Mean (SD), $\times 10^{9} / \mathrm{L}$ & 260 (99.5) & $264.0(100.9)$ & $253.3(60.1)$ & 210.6 (129.2) & 0.381 \\
\hline White blood cell, Mean (SD), $\times 10^{9} / \mathrm{L}$ & $7.3(7.9)$ & $7.7(8.5)$ & $6.0(2.2)$ & $4.0(2.5)$ & 0.412 \\
\hline Red blood cell, Mean (SD), $\times 10^{9} / \mathrm{L}$ & $4.6(0.6)$ & $4.6(0.5)$ & $4.5(0.4)$ & $3.6(0.5)$ & $<0.001$ \\
\hline Neutrophil, Mean (SD), $\times 10^{9} / \mathrm{L}$ & $3.8(4.9)$ & $3.9(5.2)$ & $4.0(2.0)$ & $1.8(1.6)$ & 0.556 \\
\hline Lymphocyte, Mean (SD), $\times 10^{9} / \mathrm{L}$ & $2.7(1.5)$ & $2.9(1.5)$ & $1.5(0.6)$ & $1.7(1.4)$ & 0.001 \\
\hline Monocyte, Mean (SD), $\times 10^{9} / \mathrm{L}$ & $0.7(2.5)$ & $0.8(2.7)$ & $0.5(0.3)$ & $0.5(0.4)$ & 0.909 \\
\hline Eosinophil, Mean (SD), $\times 10^{9} / \mathrm{L}$ & $0.1(0.2)$ & $0.1(0.2)$ & $0.0(0.0)$ & $0.0(0.0)$ & 0.008 \\
\hline Basophil, Mean (SD), $\times 10^{9} / \mathrm{L}$ & $0.0(0.0)$ & $0.0(0.0)$ & $0.0(0.0)$ & $0.0(0.0)$ & 0.373 \\
\hline Immune Factor Results & $(n=102)$ & $(n=86)$ & $(n=9)$ & $(n=7)$ & \\
\hline $\mathrm{T}$, Mean (SD), $/ \mu \mathrm{L}$ & $2260(1190)$ & 2499.0 (1113.9) & 1233.1 (562.6) & $607.7(585.9)$ & $<0.001$ \\
\hline Median [Min, Max] & $2020[146,5610]$ & $2240[984,5610]$ & $1190[543,2180]$ & $425[146,1840]$ & \\
\hline Th, Mean (SD), / $\mu \mathrm{L}$ & 1160 (708) & 1290.6 (690.8) & $590.2(249.3)$ & $349.4(308.6)$ & $<0.001$ \\
\hline Median [IQR] & 1030 [817] & 1130 [860.75] & $685[431.5]$ & 297 [298] & \\
\hline Tc, Mean (SD), $/ \mu \mathrm{L}$ & $941(604)$ & $1042.5(590.3)$ & $516.3(317.1)$ & $233.1(254.1)$ & $<0.001$ \\
\hline Median [IQR] & $252[307.5]$ & $310[306.5]$ & $117[100.5]$ & 72.0 [149] & \\
\hline NK, Mean (SD), / $\mu \mathrm{L}$ & $343(264)$ & $382.8(265.5)$ & $157.3(105.1)$ & $91.0(83.9)$ & 0.001 \\
\hline Median [IQR] & $523[474.75]$ & $566[440.5]$ & $272[272]$ & $254[452.5]$ & \\
\hline B, Mean (SD), / $\mu \mathrm{L}$ & $644(458)$ & $696.0(461.4)$ & $324.3(185.9)$ & $422.0(461.1)$ & 0.026 \\
\hline Median [IQR] & $1.24[0.688]$ & $1.24[0.563]$ & $1.12[0.8]$ & $1.86[1.72]$ & \\
\hline Treg, Mean (SD), / $\mu \mathrm{L}$ & $176(95.0)$ & $179.8(95.4)$ & $120.5(0.7)$ & $53.0(\mathrm{NA})$ & 0.297 \\
\hline Median [IQR] & $156[137.25]$ & 160 [140] & $121[1]$ & $53.0[\mathrm{NA}]$ & \\
\hline Th/Tc, Mean (SD) & $1.39(0.65)$ & $1.3(0.6)$ & $1.3(0.5)$ & $2.0(1.2)$ & 0.027 \\
\hline Median [IQR] & 4.82 [1.99] & $4.81[1.96]$ & $4.70[1.51]$ & $7.16[\mathrm{NA}]$ & \\
\hline Cytokine Results & $(n=121)$ & $(n=101)$ & $(n=12)$ & $(n=8)$ & \\
\hline IL-2, Mean (SD), pg/mL & $1.6(0.7)$ & $1.6(0.7)$ & $1.5(0.4)$ & $1.4(0.3)$ & 0.556 \\
\hline Median [IQR] & $1.47[0.445]$ & $1.47[0.44]$ & $1.38[0.445]$ & $1.40[0.61]$ & \\
\hline IL-4, Mean (SD), pg/mL & $3.1(1.8)$ & $3.1(1.9)$ & $3.2(1.3)$ & $2.7(0.8)$ & 0.819 \\
\hline Median [IQR] & $2.62[1.275]$ & $2.54[1.31]$ & $2.66[2.013]$ & $2.76[0.87]$ & \\
\hline IL-6, Mean (SD), pg/mL & $48.4(352)$ & $9.9(16.2)$ & $25.2(24.5)$ & $569.1(1337.3)$ & $<0.001$ \\
\hline Median [IQR] & $5.30[11.255]$ & $4.27[6.745]$ & $17.7[18.272]$ & $73.5[251.828]$ & \\
\hline IL-10, Mean (SD), pg/mL & $24.0(145)$ & $6.8(8.2)$ & $9.9(15.8)$ & $262.1(536.1)$ & $<0.001$ \\
\hline Median $[\mathrm{IQR}]$ & $4.62[3.795]$ & $4.50[2.87]$ & $4.92[3.11]$ & $43.5[256.065]$ & \\
\hline TNF-a, Mean (SD), pg/mL & $2.2(2.7)$ & $2.3(3.0)$ & $1.6(0.5)$ & $2.1(1.1)$ & 0.725 \\
\hline Median $[\mathrm{IQR}]$ & $1.67[0.815]$ & $1.67[0.826]$ & $1.69[0.743]$ & $1.67[1.203]$ & \\
\hline IFN- $\gamma$, Mean (SD), $\mathbf{p g} / \mathbf{m L}$ & $31.0(180)$ & $8.4(17.7)$ & $58.8(141.1)$ & $274.6(664.6)$ & $<0.001$ \\
\hline Median [IQR] & $3.71[5.7]$ & $3.45[2.745]$ & $13.2[27.215]$ & $10.8[179.778]$ & \\
\hline Other Inflammatory Factor Results & $(n=119)$ & $(n=99)$ & $(n=12)$ & $(n=8)$ & \\
\hline FERR, Mean (SD), $\mathbf{n g} / \mathbf{m L}$ & $412(1840)$ & $88.1(60.5)$ & $198(109)$ & $3230(5350)$ & $<0.001$ \\
\hline Median [IQR] & $81.8[85.80]$ & $68.1[61.8]$ & 169 [179] & 984 [4406] & \\
\hline Missing, no. (\%) & $38(31.9 \%)$ & $36(36.4 \%)$ & $2(16.7 \%)$ & $0(0 \%)$ & \\
\hline $\mathrm{PCT}$, Mean (SD), $\mathbf{n g} / \mathbf{m L}$ & $1.30(9.50)$ & $0.217(0.777)$ & $0.248(0.193)$ & $15.3(34.4)$ & $<0.001$ \\
\hline Median [IQR] & $0.060[0.128]$ & $0.055[0.05]$ & $0.185[0.225]$ & $2.10[8.335]$ & \\
\hline
\end{tabular}


Table 2 Peripheral blood indices' Results of children with SARS-COV-2 (Continued)

\begin{tabular}{|c|c|c|c|c|c|}
\hline Variables & Overall & Mild & Severe & Critical & $P$ Value \\
\hline Missing, no. (\%) & 7 (5.9\%) & $7(7.1 \%)$ & $0(0 \%)$ & $0(0 \%)$ & \\
\hline SAA, Mean (SD), mg/L & $51.5(66.8)$ & $29.1(47.1)$ & $121(61.7)$ & $198(2.60)$ & $<0.001$ \\
\hline Median [IQR] & $5.00[102.18]$ & $5.00[24.57]$ & 120 [90.38] & 198 [3.67] & \\
\hline Missing, no. (\%) & $71(59.7 \%)$ & $61(61.6 \%)$ & $4(33.3 \%)$ & $6(75.0 \%)$ & \\
\hline CRP, Mean (SD), mg/L & $10.3(16.7)$ & $6.03(8.13)$ & $36.0(32.9)$ & $22.5(20.0)$ & $<0.001$ \\
\hline Median [IQR] & $5.00[11.5]$ & $4.00[7.12]$ & $22.6[48.48]$ & $13.4[22.05]$ & \\
\hline Missing, no. (\%) & $4(3.4 \%)$ & $4(4.0 \%)$ & $0(0 \%)$ & $0(0 \%)$ & \\
\hline
\end{tabular}

significantly higher $\mathrm{Th} / \mathrm{Tc}$ cell ratio than groups I and II $(P=0.027)$.

From group I to III, there was a significant increase in IL-6, IL-10, and IFN- $\gamma$ (Table 2, Fig. 2), and FERR, SAA, and PCT (Table 2, Fig. 3), i.e., with increasing disease severity $(P<0.001)$. Although CRP was significantly higher in groups II and III than in group I $(P<0.001)$, its highest mean value was highest $(36.0 \pm$ $32.9 \mathrm{mg} / \mathrm{L}$ ) in group II.

The results of routine blood testing on admission showed a decrease from group I to III for hemoglobin $(P<0.001)$, red blood cell count $(P<0.001)$, and eosinophil count $(P=0.008)$. Groups II and III had significantly lower lymphocyte counts than group I $(P=0.001)$, with group II having the lowest count. Other routine blood parameters between the three groups were not significantly different (Table 2).
It should be noted that group III was younger, potentially including very young infants, even though the ANOVA testing did not reach statistical significance across groups (Table 3).

\section{Prognostic factors for early identification of critical COVID-19}

Next, we explored the possibility of early identification of children with critical COVID-19 using the above peripheral blood parameters as prognostic factors. PCA using the $\mathrm{R}$ package factoextra revealed a clear difference between the three groups, with more significant differences between groups I + II and group III (Fig. 4a). Therefore, groups I and II were combined into group A, and group III was designated group B for PCA and comparisons (Fig. 4b).
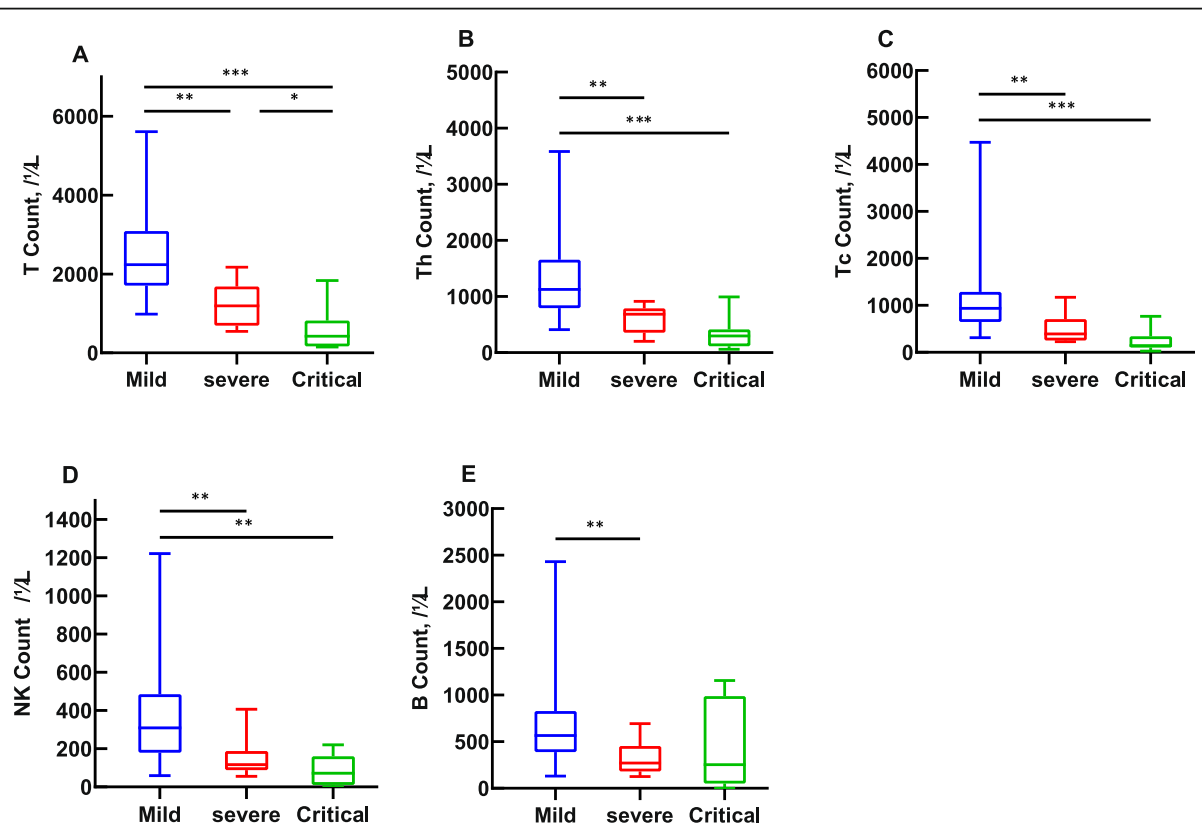

Fig. 1 Peripheral Blood Lymphocyte Subset Counts and T Cell-derived Cytokine Concentrations on Admission in Children with COVID-19. Peripheral blood lymphocyte subset counts $\left[\mathrm{CD}^{+} \mathrm{CD} 4^{+} \mathrm{T}\right.$ cells (Th), $\mathrm{CD}^{+} \mathrm{CD}^{+} \mathrm{T}$ cells $(\mathrm{Tc}), \mathrm{CD}_{16}{ }^{+} \mathrm{CD} 56^{+} \mathrm{NK}$ cells (NK), CD19+ $\mathrm{B}$ cells (B)] in the common (group I), severe (group II), and critical (group III) cases were analyzed after hospital admission. Media [min, $\max ] ;{ }^{*}, p<0.05 ;{ }^{* *}, p<0.01$, ***, $p<0.001$. Results were tested for significance with the Kruskal-Wallis test 

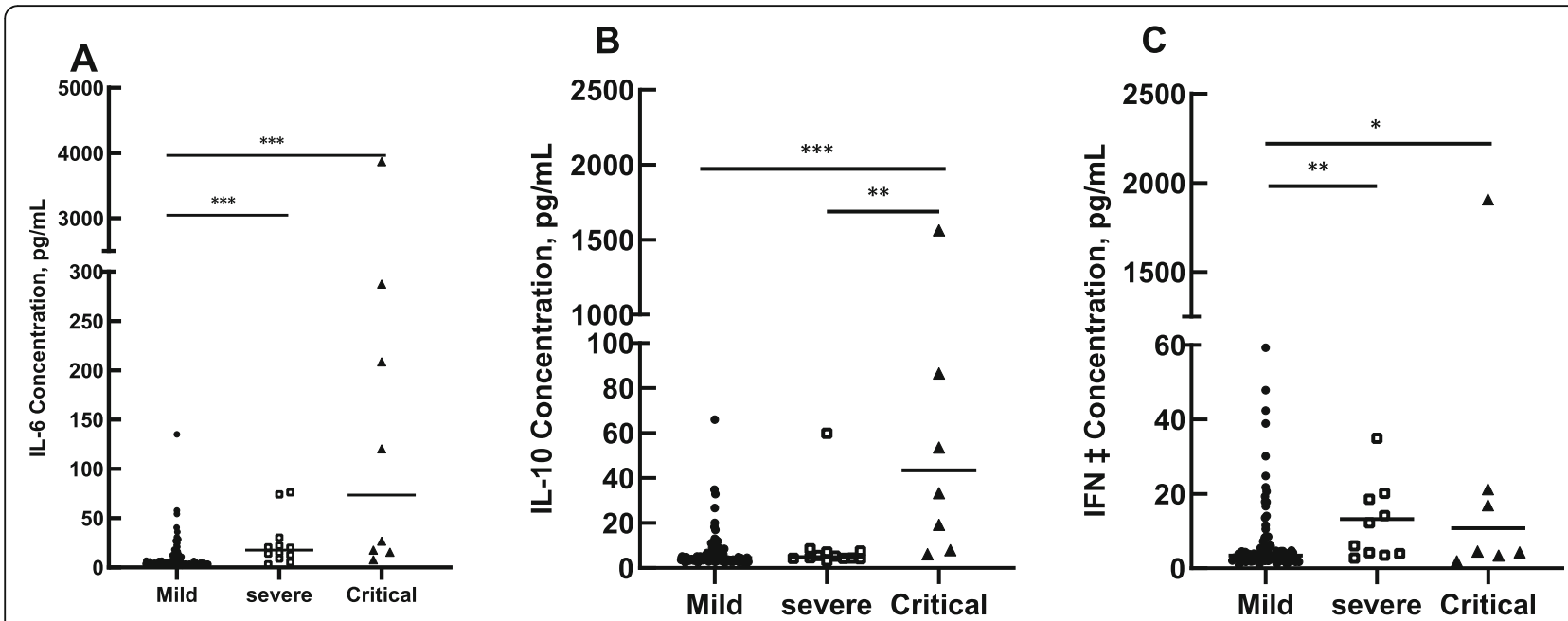

Fig. 2 T Cell-derived Cytokine Concentrations on Admission in Children with COVID-19 on Admission in Children with COVID-19. T cell-derived cytokine concentrations [a interleukin-6 (IL-6), b IL-10, c interferon- $\gamma$ (IFN- - )] in the common (group I), severe (group II), and critical (group III) cases were analyzed after hospital admission. Media [min, max]; ${ }^{*}, p<0.05 ;{ }^{* *}, p<0.01,{ }^{* * *}, p<0.001$. Results were tested for significance with the Kruskal-Wallis test

A comprehensive analysis of the contributory values suggested that 10 indices (red blood cells $[R B C]$, hemoglobin $[\mathrm{Hb}]$, lymphocytes [Lym], T lymphocytes $[T]$, Th cells $[\mathrm{Th}], \mathrm{Tc}$ cells $[\mathrm{Tc}], \mathrm{B}$ lymphocytes $[\mathrm{B}], \mathrm{IL}-6$, IL-10, IFN- $\gamma$ ) can be used as potential prognostic factors, and further statistical analysis was performed on these indices.

To determine the diagnostic value of the above 10 indices, we calculated the AUC of the ROC curve. The AUC values of $\mathrm{T}$ (0.944), RBC (0.941), Tc (0.935), IL-10

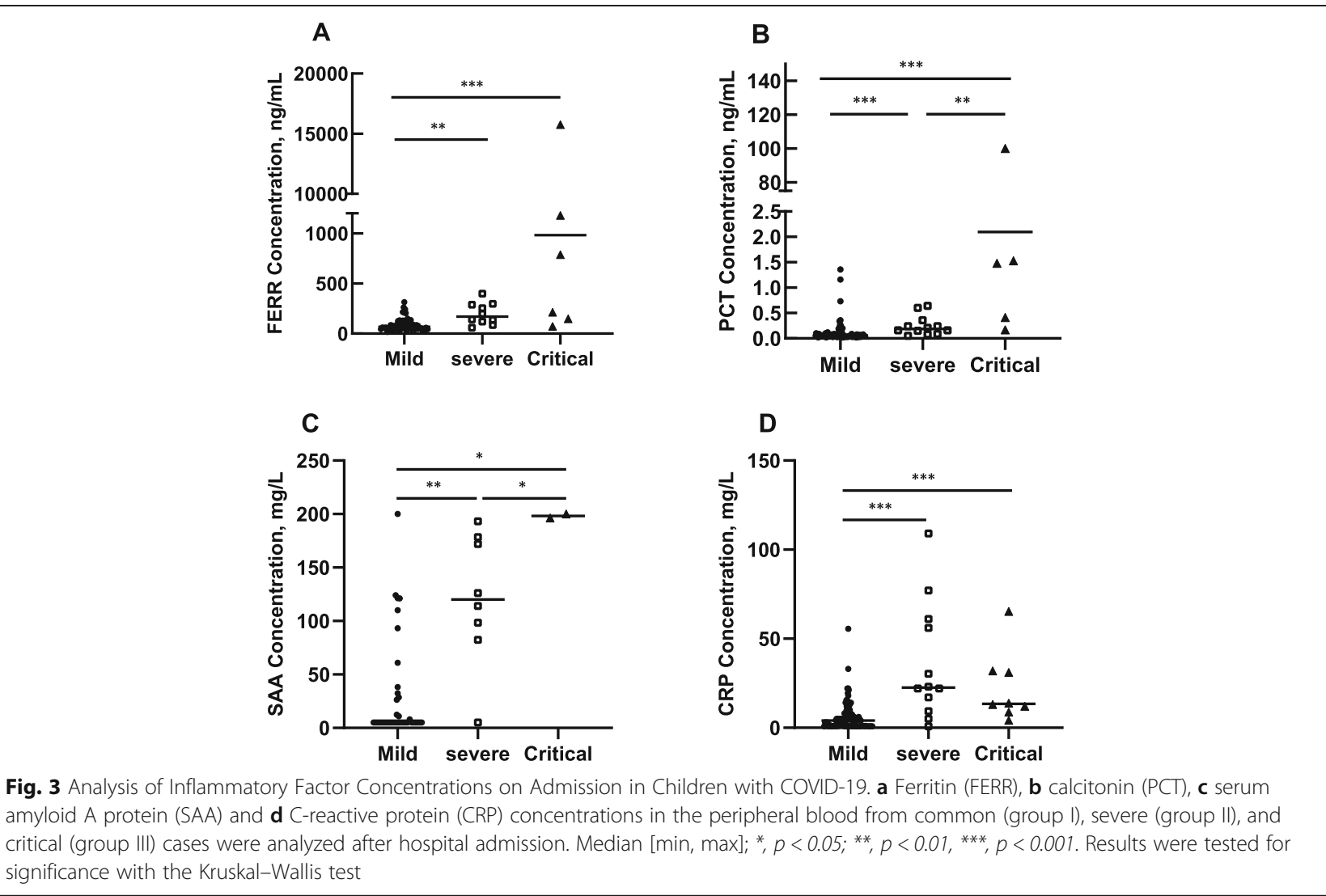


Table 3 Demographic and Clinical Features of Critical Patients

\begin{tabular}{|c|c|c|c|c|c|c|c|c|c|}
\hline & $\begin{array}{l}\text { Critical } \\
1\end{array}$ & $\begin{array}{l}\text { Critical } \\
2\end{array}$ & $\begin{array}{l}\text { Critical } \\
3\end{array}$ & $\begin{array}{l}\text { Critical } \\
4\end{array}$ & $\begin{array}{l}\text { Critical } \\
5\end{array}$ & $\begin{array}{l}\text { Critical } \\
6\end{array}$ & $\begin{array}{l}\text { Critical } \\
7\end{array}$ & $\begin{array}{l}\text { Critical } \\
8\end{array}$ & Aggregate \\
\hline Sex & Male & Male & Female & Male & Male & Male & Male & Female & 6/8 Male \\
\hline Age (years) & 6.50 & 0.17 & 0.21 & 2.58 & 1.08 & 2.25 & 8.00 & 0.87 & $\begin{array}{l}\text { Median } 1.67 \\
\text { years old }\end{array}$ \\
\hline Ventilator & No & No & Yes & No & Yes & No & Yes & Yes & $4 / 8$ \\
\hline$P C R$ & Pos & Pos & Neg & Neg & Neg & Pos & Pos & Pos & $5 / 8$ \\
\hline Imaging changes & Yes & Yes & Yes & Yes & Yes & Yes & Yes & Yes & $8 / 8$ \\
\hline Dyspnoea & No & Yes & Yes & No & Yes & Yes & No & Yes & $5 / 8$ \\
\hline Cough & Yes & Yes & Yes & No & No & Yes & Yes & No & $5 / 8$ \\
\hline Fever & Yes & Yes & Yes & Yes & Yes & Yes & Yes & Yes & $8 / 8$ \\
\hline Gastrointestinal symptoms & Yes & No & Yes & No & No & No & No & Yes & $3 / 8$ \\
\hline \multicolumn{10}{|l|}{ Comorbidities } \\
\hline Acute lymphoblastic leukemia & Yes & No & No & No & No & No & Yes & No & $2 / 8$ \\
\hline Intussusception & No & No & No & No & No & No & No & Yes & $1 / 8$ \\
\hline \multicolumn{10}{|l|}{ Complications } \\
\hline Hemophagocytic lymphohistiocytosis & No & No & No & Yes & No & No & No & No & $1 / 8$ \\
\hline Spesis & No & No & No & No & Yes & No & Yes & Yes & $3 / 8$ \\
\hline $\begin{array}{l}\text { Non-infectious multiple organ ysfunction } \\
\text { syndrome (MODS) }\end{array}$ & No & No & No & No & Yes & No & No & Yes & $1 / 8$ \\
\hline Cardiac dysfunction & No & No & No & Yes & Yes & No & Yes & No & $3 / 8$ \\
\hline Renal dysfunction & No & Yes & No & Yes & Yes & No & No & No & $3 / 8$ \\
\hline Abnormal liver function & No & Yes & No & Yes & No & No & No & No & $2 / 8$ \\
\hline Coagulation disorders & No & No & No & Yes & No & No & No & Yes & $2 / 8$ \\
\hline Epilepsy & No & No & No & No & No & No & No & Yes & $1 / 8$ \\
\hline
\end{tabular}

Demographic and clinical features of MIS-C patients $(n=8)$

(0.93), Hb (0.927), Th (0.925), and IL-6 (0.898) were greater than that of Lym (0.718), B (0.708), and IFN- $\gamma$ (0.688). Meanwhile, the cutoff values for $\mathrm{T}$ (875.5), RBC (4.195), Tc (348.5), IL-10 (6.08), Hb (117.5), Th (415.0), IL-6 (15.39), Lym (1.77), B (342.0), and IFN- $\gamma$ (4.24) were calculated according to the Youden index of the ROC curve. T, Th, Tc, IL-6, IL-10, RBC, and Hb had AUC values of $>0.85$, indicating that they contributed significantly to the identification of critical cases in this study.

\section{Discussion}

The diagnosis and treatment of severe or critical in children with COVID-19, especially infants and young children, are very different from that of adults. Symptombased clinical evaluations of children are limited by their inability to participate in examinations, essentially rendering the process of diagnosis and treatment very challenging. Therefore, determining the disease condition with the aid of laboratory tests in the early stage of SARS-CoV-2 infection in children with unspecific symptoms is very important. Children are susceptible to SARS-CoV-2; unlike adult patients, most child patients present with mild symptoms, recover quickly, and have a relatively good prognosis [1, 7]. As of February 20, 2020, a total of 12 children with severe COVID-19 and 8 children with critical COVID-19 had been admitted to our hospital, significantly fewer than the severe and critical adult cases [7-9].

In adults infected with SARS-CoV-2, the loss of $\mathrm{T}$ cells may lead to increased inflammatory response, while the recovery of $\mathrm{T}$ cell numbers helps to alleviate the degree of inflammatory response $[10,11]$. Here, we show that, in children infected with SARS-CoV-2, the laboratory examination indices of the peripheral blood in the early stage of infection had similar trends to that of adults. $\mathrm{Pa}$ tients with severe and critical COVID-19 had significantly reduced $\mathrm{T}$ lymphocyte numbers (especially $\mathrm{CD} 8^{+}$ $\mathrm{T}$ lymphocytes) and increased $\mathrm{Th} / \mathrm{Tc}$ ratios [12-15]. Children with severe disease had significantly increased inflammation-related indices (FERR, SAA, PCT) and cytokines (IL-6, IL-10, IFN- $\gamma$ ), which was contrary to the trend for $\mathrm{T}$ cells. This suggests that the immune systems, especially the immune cells, of children in the growth and development stage are quickly activated to resist the invasion and damage of SARS-CoV-2 infection. 


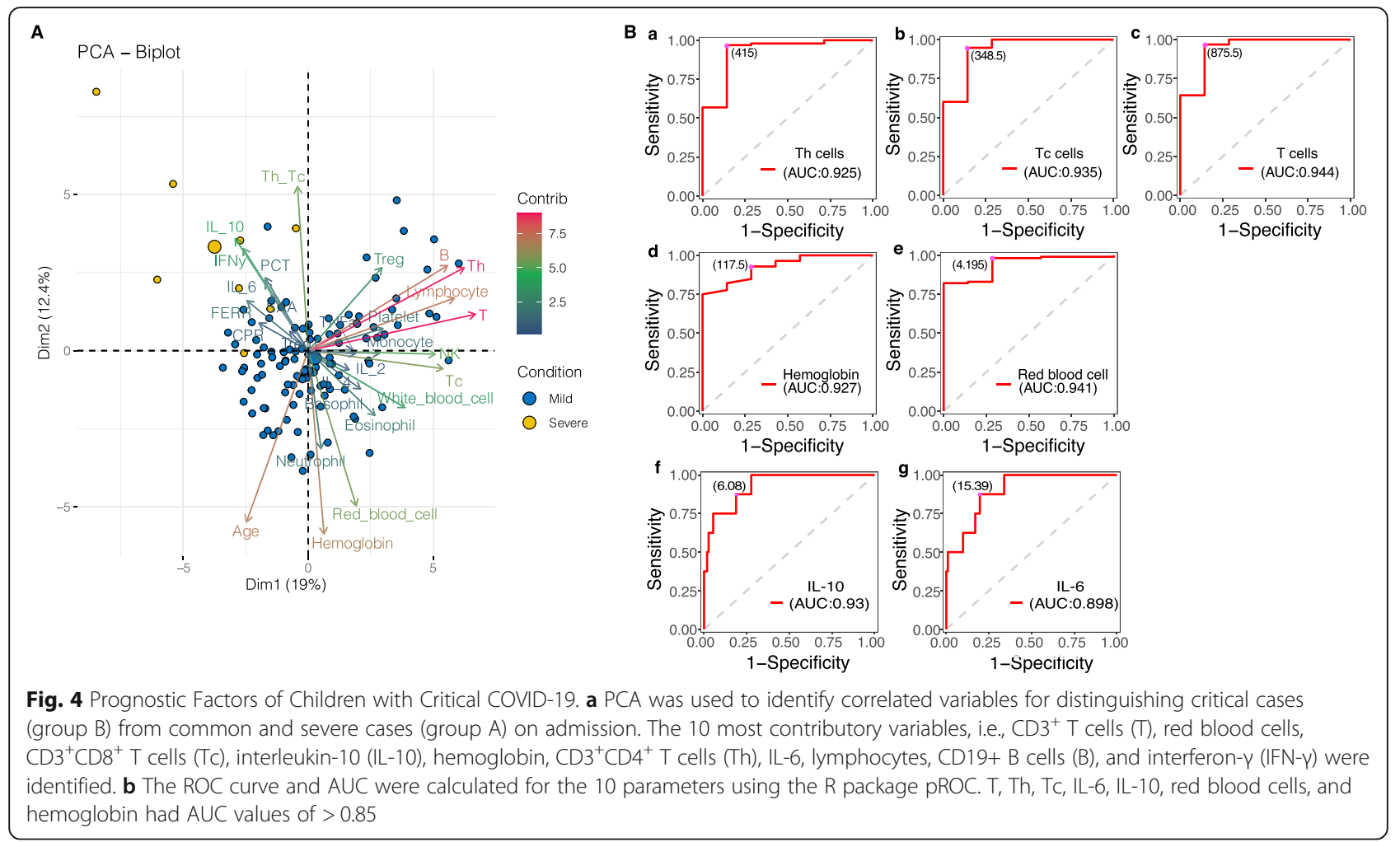

Existing reports show that adults infected with SARS$\mathrm{CoV}-2$ seemed to have inferior immune response compared to children in our study (lymphocyte: (0.6-0.9) $\times$ $10^{9} / \mathrm{L}$ in adults vs. $1.7 \times 10^{9} / \mathrm{L}$ in children), and adults with severe disease had more pronounced immune cell failure on initial admission [7-9, 11]. This may partly explain the lower proportions of severe and critical cases of SARS-CoV-2 infection in children than in adults, especially compared to the elderly $[1,7]$. However, the limited data in the present study necessitates further study of the mechanism underlying the difference between children and adults.

In the early stage of infection, influenza viruses induce IL- 6 production in the epithelial and endothelial cells of the lungs, which recruits and activates $\mathrm{CD} 8^{+}$ $\mathrm{T}$ lymphocytes and NK, Treg, and Th2 cells to secrete IFN- $\gamma$, IL-10, and IL-5 to eliminate the viruses and virus-infected cells, inhibit the exacerbation of inflammation, and restore lung function [16]. However, a sharp increase in IL-6 may be associated with poor clinical prognosis [17-20]. High IL-10 levels suggest that the body attempts to regulate the inflammatory damage caused by other actors in the cytokine storm, which indicates, to a large extent, that the inflammatory state may already be uncontrollable [21-25]. IFN- $\gamma$ has potent antiviral activity, and NK cells produce IFN $-\gamma$ in the early stage of viral infection (3-5 days after infection) to inhibit viral replication [26].
Carl A. Pierce et.al [27] reported that The neutralizing antibody concentration was higher in infected adults than in children, and the neutralizing antibody titer was inversely correlated with the serum IL-17A and IFN- $\gamma$ concentrations. In children, early immune responses mediated by cells that produce IL-17A and IFN- $\gamma$ lead to more rapid resolution of viral infection and may have mitigated against the progressive cytokine release and tissue pathology that occurs with more robust adaptive immune responses, which means the boosted early innate immune responses may be important.

Here, the IL-6, IL-10, and IFN- $\gamma$ initially tested on admission increased from group I to group III, with groups II and III having significantly higher values than group I $(P<0.001)$. This suggests that, in the children with severe and critical disease, IL-6, IL-10, and IFN- $\gamma$ had been produced to resist viral invasion and there had been attempts to suppress the inflammatory response in the early stage of the SARS-CoV2 infection.

The three cytokines were significantly higher in group III than in groups I and II, but group III had significantly lower Th and Tc cell counts than groups I and II, indicating that relatively severe inflammatory damage had occurred in the early stage of SARS-CoV-2 infection. The acute inflammatory indices, i.e., FERR, SAA, and PCT, were also significantly higher in group III than in 
groups I and II, indicating that the disease conditions of the children in group III had deteriorated rapidly, their immune system had become dysregulated, and cytokine storm was inevitable.

We found that the children in group II had relatively good treatment outcomes and similar prognoses to the children in group I, and no case progressed from group II to group III, which suggests that the pathogenesis of critical COVID-19 in children warrants further research.

We further classified the children with critical disease as group B and combined groups I and II into group A. PCA and ROC curve analysis of the two groups revealed that the indices $\mathrm{T}(0.944), \mathrm{RBC}(0.941), \mathrm{Tc}(0.935), \mathrm{IL}-10$ (0.93), Hb (0.927), Th (0.925), and IL-6 (0.898) had relatively larger AUC values, with cutoff values of 875.5, $4.195,348.5,6.08,117.5,415.0$, and 15.39 , respectively. This suggests that the seven indices are of great contribution to the early identification and prediction of the prognosis of children with critical COVID-19.

Mild patients in this study did not have typical multisystem inflammatory syndrome in children (MIS-C) phenotypes. Only one critical child appeared to have MIS-C phenotypes, according to Gruber CN PR, Camila Rosat Consiglio et.al, $[28,29]$ during the treatment process. Follow-up 4 months later was showed that peripheral blood indexes were almost normal, but abnormal liver, kidney, myocardium and coagulation function remained.

In short, as children are susceptible to SARS-CoV-2, it is particularly important to preliminarily distinguish between mild and severe disease through simple testing at the initial stage of admission. Our study suggests that seven indices ( $T$, Th, Tc, IL-6, IL-10, RBC, Hb) are helpful for early diagnosis of COVID-19 severity in children, and may provide a laboratory basis for clinicians to offer timely treatment to children with critical COVID-19. Due to the limited sample size, further multi-center, large-sample studies are needed.

\section{Code availability}

Not applicable.

\section{Authors' contributions}

Hao Xiong and Wenjie Lu had full access to all of the data in the study and take responsibility for the integrity of the data and the accuracy of the data analysis. The first draft of the manuscript was written by Wenjie Lu, Xiong Li, Hao Xiong and all authors commented on previous versions of the manuscript. All authors read and approved the final manuscript. Wenjie Lu, Li Yang and Xiong Li contributed equally and share first authorship. Concept and design: Wenjjie Lu, Li Yang, Xiong Li, Hao Xiong; Acquisition, analysis, or interpretation of data: Wenjie Lu, Li Yang, Xiong Li, Aiping Zhang, Shanshan Qi, Ming Sun, Zhi Chen, Lannan Zhang, Jianxin Li, Hao Xiong: Drafting of the manuscript: Wenjie Lu, Li Yang, Xiong Li, Hao Xiong; Critical revision of the manuscript for important intellectual content: Wenjie $L u, L i$ Yang, Xiong Li, Aiping Zhang, Shanshan Qi, Ming Sun, Zhi Chen, Lannan Zhang, Jianxin Li, Hao Xiong; Statistical analysis: Wenjie Lu, Xiong Li, Li Yang, Hao Xiong. Role of the Funder/Sponsor: The funders had no role in the design and conduct of the study; collection, management, analysis, and interpretation of the data; preparation, review, or approval of the manuscript; and decision to submit the manuscript for publication.

\section{Funding}

The National Natural Science Foundation of China (81902653) and Wuhan Municipal health Commission (WX18Q16).

\section{Availability of data and materials}

The datasets generated during and/or analyzed during the current study are available from the corresponding author on reasonable request. Patients have not been reported in any other submission by anyone.

\section{Ethics approval and consent to participate}

Ethical approval was waived by the local Ethics Committee of Huazhong University of Science and Technology in view of the retrospective nature of the study and all the procedures being performed were part of the routine care.

Informed consent was obtained from all individual participants included in the study and all the experiments were performed in accordance with relevant guidelines and regulations.

\section{Consent for publication}

Not applicable.

\section{Competing interests}

All the authors have no conflict of interest.

\section{Author details}

${ }^{1}$ Department of Hematology, Wuhan Children's Hospital (Wuhan Medical Care Center for Women and Children), Tongji College, Huazhong University of Science and Technology, Wuhan, Hubei, China. 'Department of Surgery, University of Michigan School of Medicine, Ann Arbor, MI, USA. ${ }^{3}$ Center of Excellence for Cancer Immunology and Immunotherapy, University of Michigan Rogel Cancer Center, University of Michigan School of Medicine, Ann Arbor, MI, USA.

Received: 16 October 2020 Accepted: 15 February 2021

Published online: 17 April 2021

\section{References}

1. Lu X, Zhang L, Du H, et al. SARS-CoV-2 Infection in Children. N Engl J Med. 2020;382(17):1663-5.

2. Behrens EM, Koretzky GA. Review: cytokine storm syndrome: looking toward the precision medicine era. Arthritis Rheum. 2017:69(6):1135-43.

3. Tisoncik JR, Korth MJ, Simmons CP, Farrar J, Martin TR, Katze MG. Into the eye of the cytokine storm. Microbiol Mol Biol Rev. 2012;76(1):16-32.

4. Henter Jl, Chow CB, Leung CW, Lau YL. Cytotoxic therapy for severe avian influenza a (H5N1) infection. Lancet. 2006:367(9513):870-3.

5. Society of Pediatrics CMA. Editorial board CJOP: recommendations for the diagnosis, prevention and control of the 2019 novel coronavirus infection in children (first interim edition). Zhonghua Er Ke Za Zhi. 2020;58(3):169-74.

6. World Health Organization. Country \& technical guidance - coronavirus disease (COVID-19). https://www.who.int/emergencies/diseases/novelcoronavirus-2019/technical-guidance. Accessed 17 July 2020.

7. Guan WJ, Ni ZY, Hu Y, et al. Clinical Characteristics of Coronavirus Disease 2019 in China. N Engl J Med. 2020;382(18):1708-20.

8. Wang D, Hu B, Hu C, et al. Clinical Characteristics of 138 Hospitalized Patients With 2019 Novel Coronavirus-Infected Pneumonia in Wuhan, China. JAMA. 2020:323(11):1061-9. https://doi.org/10.1001/jama.2020.1585.

9. Chen N, Zhou M, Dong X, Qu J, Gong F, Han Y, Qiu Y, Wang J, Liu Y, Wei Y, et al. Epidemiological and clinical characteristics of 99 cases of 2019 novel coronavirus pneumonia in Wuhan, China: a descriptive study. Lancet. 2020; 395(10223):507-13.

10. Kim KD, Zhao J, Auh S, Yang X, Du P, Tang H, Fu YX. Adaptive immune cells temper initial innate responses. Nat Med. 2007;13(10):1248-52.

11. Liu J, Li S, Liu J, et al. Longitudinal characteristics of lymphocyte responses and cytokine profiles in the peripheral blood of SARS-CoV-2 infected patients. EBioMed. 2020;55:102763.

12. Huang C, Wang Y, Li X, Ren L, Zhao J, Hu Y, Zhang L, Fan G, Xu J, Gu X, et al. Clinical features of patients infected with 2019 novel coronavirus in Wuhan, China. Lancet. 2020;395(10223):497-506. 
13. Xu Z, Shi L, Wang Y, Zhang J, Huang L, Zhang C, Liu S, Zhao P, Liu H, Zhu L, et al. Pathological findings of COVID-19 associated with acute respiratory distress syndrome. Lancet Respir Med. 2020;8(4):420-2.

14. Qin C, Zhou L, Hu Z, Zhang S, Yang S, Tao Y, Xie C, Ma K, Shang K, Wang W, et al. Dysregulation of immune response in patients with COVID-19 in Wuhan. Clin Infect Dis: China; 2020.

15. Shi Y, Tan M, Chen X, Liu Y, Huang J, Ou J, Deng X. Immunopathological characteristics of coronavirus disease 2019 cases in Guangzhou, China. Preprint at medRxiv. 2020. https://doi.org/10.1101/2020031220034736

16. Longhi MP, Wright $K$, Lauder SN, et al. Interleukin-6 is crucial for recall of influenza-specific memory CD4 T cells. PLoS Pathog. 2008;4(2):e1000006.

17. Cao X. COVID-19: immunopathology and its implications for therapy. Nat Rev Immunol. 2020;20(5):269-70.

18. Oshansky CM, Gartland AJ, Wong SS, Jeevan T, Wang D, Roddam PL, Caniza MA, Hertz T, Devincenzo JP, Webby RJ, et al. Mucosal immune responses predict clinical outcomes during influenza infection independently of age and viral load. Am J Respir Crit Care Med. 2014;189(4):449-62.

19. Kaiser L, Fritz RS, Straus SE, Gubareva L, Hayden FG. Symptom pathogenesis during acute influenza: interleukin-6 and other cytokine responses. J Med Virol. 2001;64(3):262-8.

20. Hagau N, Slavcovici A, Gonganau DN, Oltean S, Dirzu DS, Brezoszki ES, Maxim M, Ciuce C, Mlesnite M, Gavrus RL, et al. Clinical aspects and cytokine response in severe H1N1 influenza a virus infection. Crit Care. 2010; 14(6):R203.

21. Couper KN, Blount DG, Riley EM. IL-10: the master regulator of immunity to infection. J Immunol. 2008:180(9):5771-7.

22. Ouyang W, Rutz S, Crellin NK, Valdez PA, Hymowitz SG. Regulation and functions of the IL-10 family of cytokines in inflammation and disease. Annu Rev Immunol. 2011;29:71-109.

23. Saraiva M, O'Garra A. The regulation of IL-10 production by immune cells. Nat Rev Immunol. 2010;10(3):170-81.

24. de Jong MD, Simmons CP, Thanh TT, Hien VM, Smith GJ, Chau TN, Hoang DM, Chau NV, Khanh TH, Dong VC, et al. Fatal outcome of human influenza a (H5N1) is associated with high viral load and hypercytokinemia. Nat Med. 2006;12(10):1203-7.

25. Shen H-H, Hou J, Chen W-W, Bai B-K, Wang H-B, Guo T-S, Liu A-X, Li Y-L, Zhao M, Mao P-Y, et al. Immunologic changes during pandemic (H1N1) 2009, China. Emerg Infect Dis. 2011;17(6):1053-5.

26. Guo XJ, Thomas PG. New fronts emerge in the influenza cytokine storm. Semin Immunopathol. 2017;39(5):541-50.

27. Pierce CA, Preston-Hurlburt P, Dai Y, et al. Immune responses to SARS-CoV-2 infection in hospitalized pediatric and adult patients. Sci Transl Med. 2020; 12(564):eabd5487.

28. Gruber CN, Patel RS, Trachtman R, et al. Mapping Systemic Inflammation and Antibody Responses in Multisystem Inflammatory Syndrome in Children (MIS-C). Cell. 2020;183(4):982-995.e14.

29. Consiglio CR, Cotugno N, Sardh F, Pou C, Amodio D, Rodriguez L, Tan Z, Zicari S, Ruggiero A, Pascucci GR, Santilli V, Campbell T, Bryceson Y, Eriksson D, Wang J, Marchesi A, Lakshmikanth T, Campana A, Villani A, Rossi P, the CACTUS Study Team, Landegren N, Palma P, Brodin P. The Immunology of Multisystem Inflammatory Syndrome in Children with COVID-19. Cell. 2020

\section{Publisher's Note}

Springer Nature remains neutral with regard to jurisdictional claims in published maps and institutional affiliations.

Ready to submit your research? Choose BMC and benefit from:

- fast, convenient online submission

- thorough peer review by experienced researchers in your field

- rapid publication on acceptance

- support for research data, including large and complex data types

- gold Open Access which fosters wider collaboration and increased citations

- maximum visibility for your research: over $100 \mathrm{M}$ website views per year

At $\mathrm{BMC}$, research is always in progress.

Learn more biomedcentral.com/submissions 